\title{
Clinical outcomes and risk factors of secondary extraintestinal manifestation in ulcerative colitis: Results of a multicenter and long-term follow-up retrospective study
}

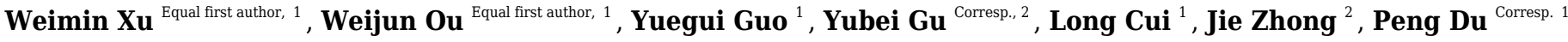 \\ 1 Department of Colorectal Surgery, Xin-Hua Hospital, Shanghai Jiaotong University School of Medicine, Shanghai, China \\ 2 Department of Gastroenterology, Rui Jin Hospital, affiliate to Shanghai Jiao Tong University, School of Medicine, Shanghai, China \\ Corresponding Authors: Yubei Gu, Peng Du \\ Email address: gyb11809@rjh.com.cn, dupeng@xinhuamed.com.cn
}

Background: Extraintestinal manifestations (EIM) are common in ulcerative colitis (UC). In Shanghai, China, data on the incidence rate and risk factors of EIM in UC patients remain scarce. Methods: The study population consisted of UC patients who were identified from a prospectively maintained, institutional review board-approved database at our institutes from June 1986 to December 2018. The demographic and clinical characteristics of the study participants were analyzed. The study included secondary EIM in UC patients and follow-up, while primary EIM was excluded. The diagnosis of EIM was based on clinical, radiological, endoscopic, and immunologic examination and histological findings. Results: In total, 271 eligible patients were included in the current study, with a median follow-up time of 13.0 years [interquartile range (IQR), 9.0-17.0], and including 31 cases (11.4\%) that developed EIM. EIM was associated with clinical outcomes in UC patients and the following factors were identified as contributing factors for the development of EIM: a disease duration of $>5$ years [odds ratio (OR), 3.721; 95\% confidence interval $(\mathrm{Cl})$, 1.209-11.456], age at diagnosis $>40$ years (OR, 2.924, 95\% Cl, 1.165-7.340), refractory clinical symptoms (OR, 4.119; 95\% Cl, 1.758-9.650), and moderate or severe anemia (OR, 2.592; $95 \% \mathrm{Cl}, 1.047-6.413)$. Conclusion: In this study, approximately $11.4 \%$ UC patients go on to develop at least one EIM. Clinicians should prioritize early control of the disease and treatment of anemia in UC in order to prevent the development of EIM and improve disease prognosis. 
1 Clinical outcomes and risk factors of secondary extraintestinal manifestation in ulcerative

2

3 Weimin $\mathrm{Xu}^{1 \#}$, Weijun $\mathrm{Ou}^{1 \#}$, Yuegui Guo ${ }^{1}$, Yubei $\mathrm{Gu}^{2 *}$, Long Cui ${ }^{1}$, Jie Zhong ${ }^{2}$, Peng Du ${ }^{*}$

$4{ }^{1}$ Department of Colorectal Surgery, Xin-Hua Hospital, Shanghai Jiaotong University School of

5 Medicine, Shanghai, China

$6{ }^{2}$ Department of Gastroenterology, Rui Jin Hospital, affiliate to Shanghai Jiao Tong University,

7 School of Medicine, Shanghai, China.

8

9 Weimin $\mathrm{Xu}$ and Weijun Ou contributed equally to this work.

11 Corresponding author:

12 Peng Du, MD, Department of Colorectal Surgery, Xin-Hua Hospital, Shanghai, China. Email:

13 dupeng@xinhuamed.com.cn; Tel: 086-021-25077858;

14 Yubei Gu, MD, Department of Gastroenterology, Rui Jin Hospital, affiliate to Shanghai Jiao Tong

15 University, School of Medicine, Shanghai, China. Email: gyb11809@rjh.com.cn. 


\section{Abstract}

Background: Extraintestinal manifestations (EIM) are common in ulcerative colitis (UC). In Shanghai, China, data on the incidence rate and risk factors of EIM in UC patients remain scarce.

Methods: The study population consisted of UC patients who were identified from a prospectively maintained, institutional review board-approved database at our institutes from June 1986 to December 2018. The demographic and clinical characteristics of the study participants were analyzed. The study included secondary EIM in UC patients and follow-up, while primary EIM was excluded. The diagnosis of EIM was based on clinical, radiological, endoscopic, and immunologic examination and histological findings.

Results: In total, 271 eligible patients were included in the current study, with a median followup time of 13.0 years [interquartile range (IQR), 9.0-17.0], and including 31 cases (11.4\%) that developed EIM. EIM was associated with clinical outcomes in UC patients and the following factors were identified as contributing factors for the development of EIM: a disease duration of $>$ 5 years [odds ratio (OR), 3.721; 95\% confidence interval (CI), 1.209-11.456], age at diagnosis > 40 years (OR, 2.924, 95\% CI, 1.165-7.340), refractory clinical symptoms (OR, 4.119; 95\% CI, 1.758-9.650), and moderate or severe anemia (OR, 2.592; 95\% CI, 1.047-6.413).

Conclusion: In this study, approximately $11.4 \%$ UC patients go on to develop at least one EIM. Clinicians should prioritize early control of the disease and treatment of anemia in UC in order to prevent the development of EIM and improve disease prognosis.

\section{Introduction}

As a major form of inflammatory bowel disease (IBD), ulcerative colitis (UC) is a multifactorial 
40 polygenic disease with probable genetic heterogeneity, which is characterized by a chronic course

41 of recurrent relapse and remission (Fiocchi 1998; Podolsky 2002; SC 2017). In clinical practice,

UC patients often present with complex and diverse gastrointestinal symptoms combined with

43 relatively rare extraintestinal manifestations (EIM). The clinical presentation of EIM is extremely

heterogeneous and can affect almost all of the organs in the body; EIM is especially involved in

blood, joints, skin, eyes, biliary tracts, the vascular system, and organs such as the liver, kidneys,

and lungs. Some diseases, such as oral aphthous ulcers and erythema nodosum, parallel the activity

of the bowel disease, but for a number of these conditions, such as ankylosing spondylitis, they

follow an independent course from the disease activity of UC (Rothfuss et al. 2006; Trikudanathan

et al. 2012). All these clinical symptoms refer to the secondary EIM, while primary EIM refers to

extraintestinal symptoms that occur before the onset of UC and are not related to UC.

EIM affects the outside gastrointestinal tract and some forms are associated with $\mathrm{UC}$ as a result of autoimmune diseases such as rheumatoid arthritis (RA) and vasculitis; these diseases can sometimes mimic each other, making differential diagnosis difficult for clinicians (Colia et al. 2016). Moreover, multiple EIM may occur concomitantly, and the presence of a single EIM confers a higher likelihood of developing additional EIM (Vavricka et al. 2011), which seriously compromises the patients' quality of life and aggravates the disease. Therefore, early diagnosis and prompt intervention are imperative for EIM in clinical practice. However, few studies have specifically reported the clinical outcomes, especially the risk factors, of EIM in the Chinese population.

In the present study, we mainly research the incidence rate of EIM in Shanghai city and the associations between EIM and clinical outcomes. Additionally, we also aim to determine the risk 
62 factors contributing to the development of EIM, which could aid in earlier identification, and 63 further prevent the development of EIM.

64 


\section{Methods}

\section{Study population}

All consecutive UC patients at the Department of Colorectal Surgery, Xin-Hua Hospital, Shanghai Jiao Tong University School of Medicine (Shanghai, China), and the Department of Gastroenterology, Rui-Jin Hospital, Shanghai Jiao Tong University School of Medicine (Shanghai, China) from June 1986 to December 2018 were enrolled in the study. Patients were identified and their clinical data was obtained from a prospectively maintained, institutional review board-approved database (Chinese Database System for IBD, CHASE-IBD). The Ethics Committee of Xin-hua Hospital approved this study (approval no. XHEC-D-2018-089).

\section{Inclusion and exclusion criteria}

Inclusion criteria were as follows: (1) positive diagnosis of UC; (2) age at diagnosis $\geq 18$ years old; and (3) received a regular follow-up at our department. Exclusion criteria were as follows: (1) patients who were diagnosed with familial adenomatous polyposis (FAP) or indeterminate colitis (IC); (2) patients with poor compliance; and (3) patients with underlying disease, impaired general health, and/or lost to follow-up.

In this study, poor patient compliance refers to patients who refuse to receive regular follow-up and give feedback to the doctor. Underlying disease was mainly defined as the infectious factors caused of intestinal inflammation as well as colorectal cancer (CRC) or high-grade dysplasia. The impaired general health refers to serious and chronic diseases such as hypertension, diabetes, hyperthyroidism and heart failure, which lead to poor general conditions.

\section{Data collection and clinical evaluation}


The patient's medical history, examination data, surgical information, and clinical outcomes were retrospectively collected from the hospital medical records, outpatient examination, and long-term regular follow-up. The collected data were as follows: sex, age at diagnosis, disease duration, relapse, EIM, gastrointestinal symptoms, weight loss, primary sclerosing cholangitis (PSC), disease extent according to the Montreal classification system(Silverberg et al. 2005), surgical history, familial history of IBD or CRC, complications, development of CRC, use of steroids and immunomodulators, and hemoglobin $(\mathrm{Hb})$ and Albumin (Alb) measurements. Each patient will undergo routine and systematic imaging examinations, colonoscopy or biopsy, immunological examinations, etc. All these baseline characteristics were presented based on the most recent follow-up. Relevant data of EIM was collected during treatment. In addition, the use of mesalamine, biologics, steroids and immunomodulators refer to current treatment or the previous treatment that has been used until the latest follow-up (December 2018). UC was strictly diagnosed according to the established internationally accepted criteria proposed by Lennard-Jones(LennardJones 1989). The criteria excluded infectious factors caused of intestinal inflammation. The diagnosis of UC and EIM were based on clinical, radiological, endoscopic, and immunologic examination, and histologic findings. In this study, primary EIM refers to extraintestinal symptoms that occur before the onset of UC and are not related to UC. While secondary EIM refers to extraintestinal symptoms that occur in the course of UC that affect various organs in the body. It includes various clinical manifestations such as rash, oral aphthous ulcers, arthritis, glomerulonephritis, PSC, vasculitis, ankylosing spondylitis and autoimmune hepatitis. Only when these extraintestinal symptoms appear in the course of UC, and after detailed radiological, immunologic and histologic examination, can be diagnosed as a secondary EIM. The rash in the text refers to the long-term persistent skin damage that occurs in the course of UC, while the 
111 transient rash that occurs after drug treatment is not taken into account. We defined refractory

112 clinical symptoms as more than 5 stools per day accompanied by diarrhea and mucous, and bloody

113 stools after regular medical treatment, including 5-aminosalicylic acids (5-ASA), corticosteroid,

114 immunomodulators, and biologics. In this study, patients with 'weight loss' refers to patients who

115 lost more than $5 \mathrm{~kg}$ at the latest follow-up. Family history of other cancers refers to first, second

116 or third degree relative of the patient had or is currently living with cancer except CRC. Mild

117 anemia was defined as $\mathrm{Hb}$ value was less than $120 \mathrm{~g} / \mathrm{L}$ but more than $90 \mathrm{~g} / \mathrm{L}$ in male and less than

$118110 \mathrm{~g} / \mathrm{L}$ but more than $90 \mathrm{~g} / \mathrm{L}$ in female. Moderate anemia refers to $\mathrm{Hb}$ value between $60 \mathrm{~g} / \mathrm{L}$ and

$11990 \mathrm{~g} / \mathrm{L}$, and severe anemia was defined as $\mathrm{Hb}$ value $<60 \mathrm{~g} / \mathrm{L}$ both in male and female, respectively.

120 Therefore, in this study, we modified the definition that $\mathrm{Hb}<90 \mathrm{~g} / \mathrm{L}$ refers to moderate or severe

121 anemia. Patients with $\mathrm{Hb}$ value $<90 \mathrm{~g} / \mathrm{L}$ has at least a moderate anemia even more serious anemia.

122 Remission in this study refer to the relief of clinical symptoms including less than 3 times of stools

123 per day, the disappearance of diarrhea, fever, abdominal bloating and distension, and stool with

124 mucus, pus and blood. To evaluate the associations between EIM and clinical outcomes, we

125 divided the patients into two groups with or without EIM. The complications refer to various

126 clinical manifestations as well as the development of CRC. All complications were diagnosed

127 based on clinical manifestations, laboratory results, and endoscopic and imaging findings.

128 Intestinal obstruction, colon perforation and gastrointestinal bleeding were defined as the serious

129 complications.

130

131 Statistical analysis

132 SPSS version 19.0 software (IBM Corp., Armonk, NY, USA) were used for the statistical

133 analyses. Values and percentages, mean and standard deviations (SD), or the median and 
134 interquartile range (IQR) were used to describe the different varieties of data, respectively. Chi-

135 squared or Fisher's exact test were used for categorical variables. Moreover, we choose the

136 Wilcoxon's rank-sum test as the statistical method for ranked variables. Multivariate logistic

137 regression was performed in the variables with $\mathrm{p}$ value $<0.05$ in the analysis of the risk factors

138 of EIM in UC. Confidence intervals (CI) were set at 95\%. All statistical tests were two-sided,

139 with a p-value of $<0.05$ considered statistically significant.

140 
141

142

143

144

145

146

147

148

149

150

151

152

153

154

155

156

157

158

159

160

161

162

163

\section{Results}

\section{Demographics and clinical characteristics}

A total of 291 UC patients were enrolled at our institute, 20 patients were lost to follow-up and excluded; 271 eligible patients were ultimately included for the current research. A schematic flow diagram of the present study is shown in Figure 1 and the patient demographics and clinical and laboratory characteristics are shown in Table 1. In the entire cohort, the median follow-up time was 13.0 years (IQR: 9.0-17.0 years) from June 1986 to December 2018. Among the 271 patients, 132 were males and patients had a median age at diagnosis of 42.0 years (IQR: 29.0-53.3 years) and a median disease duration of 7.0 years (IQR: 4.0-10.0 years). In the entire cohort, 31 patients (11.4\%) were found to develop at least one EIM, of which 8 patients (3.0\%) developed multiple EIM. In addition, 6 patients $(2.2 \%)$ had proctitis (E1), $140(51.7 \%)$ had left-sided colitis (E2), and $125(46.1 \%)$ had pancolitis (E3). In addition, in the total of 31 EIM patients, 14 were men. Median age at diagnosis and disease duration in EIM patients was 47.0 years (IQR: 44.0-55.0 years) and 14,0 years (IQR:6.0-17.0 years), respectively (Table 1).

5

2. Analysis of EIM in the present study

Table 2 showed the detailed EIM in UC patients. In the 23 patients with single EIM, 9 patients developed arthritis, 6 had oral aphthous ulcers, 4 had rash, 2 experienced glomerulonephritis, 1 had PSC and 1 had ankylosing spondylitis. For 8 patients with multiple EIM, 3 patients had arthritis and oral aphthous ulcers, 1 had arthritis and rash, 1 had oral aphthous ulcers and rash, 1 had arthritis and iritis, 1 developed rash and vasculitis and 1 experienced PSC and autoimmune hepatitis (Table 2). Therefore, this data indicated that the overall incidence rate of EIM of UC in Shanghai was $11.4 \%$ 


\section{Analysis of complications in UC}

166

167

168

In the entire cohort, 107 patients (39.5\%) had complications during hospitalization or follow-up. The most common complication was inflammatory polyps, which occurred in 63 patients (23.2\%). In addition, 28 (10.3\%) experienced gastrointestinal bleeding, 21 (7.7\%) demonstrated intestinal obstruction and $6(2.2 \%)$ had colon perforation. Intestinal obstruction, colon perforation and gastrointestinal bleeding were defined as the serious complications. Moreover, 11 patients (4.1\%) went on display malignant transformation and were subsequently diagnosed with CRC. In EIM patients, 8 patients $(25.8 \%)$ developed colorectal stricture, $5(16.1 \%)$ had intestinal obstruction and $4(12.9 \%)$ had CRC (Table 3).

\section{Comparison of clinical outcomes between UC patients with and without EIM}

Clinical outcomes including remission, serious complications (intestinal obstruction or perforation or bleeding), surgery, development of CRC, and death were analyzed in Non-EIM group and EIM group. As shown in Table 4, remission was more common in the non-EIM group $(\mathrm{n}=127,52.9 \%)$ than in the EIM group $(n=8,25.8 \%)(p=0.004)$. Patients with EIM had a higher likelihood of developing serious complications than those in the non-EIM group $(n=10,32.3 \%$ vs $n=40$, $16.7 \%, \mathrm{p}=0.035)$. In addition, patients in the EIM group had a higher risk of developing CRC than those in the non-EIM group $(n=4,12.9 \%$ vs $n=7,2.9 \%, p=0.030)$. There were no statistical differences between the two groups with regards to cure, disease activity, surgery, or death. Taken together, this result suggested that EIM was closely associated with clinical outcomes in UC.

\section{Risk factors for the development of EIM}


187 Based on the previous results, we determined which risk factors were associated with the 188 development of EIM. The results of univariable analysis showed that the age at diagnosis $(\mathrm{p}=$ $1890.011)$, disease duration $(\mathrm{p}=0.013)$, refractory clinical symptoms $(\mathrm{p}<0.001)$, and the presence of 190 moderate or severe anemia $(\mathrm{p}=0.024)$ were all significantly associated with the development of 191 EIM. No significant differences were observed between the EIM group and the non-EIM group in 192 terms of sex, relapse, weight loss, history of surgery, family history, extent of UC, use of steroids 193 and immunomodulators, and the Alb level (Table 5). The results of multivariate logistic analysis 194 demonstrated that a disease duration $>5$ years $(\mathrm{OR}, 3.721 ; 95 \% \mathrm{CI}, 1.209-11.456 ; \mathrm{p}=0.022)$, an 195 age at diagnosis $>40$ years $(\mathrm{OR}, 2.924,95 \% \mathrm{CI}, 1.165-7.340 ; \mathrm{p}=0.022)$ and refractory clinical 196 symptoms (OR, 4.119; 95\% CI, 1.758-9.650; $\mathrm{p}=0.001)$ were associated with the development of 197 EIM. In addition, moderate or severe anemia $(\mathrm{OR}, 2.592 ; 95 \% \mathrm{CI}, 1.047-6.413 ; \mathrm{p}=0.039)$ was 198 associated with the development of EIM (Table 6). 
199

200

201

202

203

204

205

206

207

208

209

210

211

212

213

214

215

216

217

218

219

220

221

\section{Discussion}

It is a challenge for clinicians to accurately diagnose EIM due to the complexity and diversity of its clinical manifestations. Although EIM is relatively rare and more frequently ignored compared to the gastrointestinal symptoms of $\mathrm{UC}$, it tends to aggravate the severity of disease leading to a poor prognosis. There are a limited number of studies that have researched the effect of EIM on clinical outcomes, especially the risk factors for the development of EIM in the Chinese UC population. To the best of our knowledge, this study is the largest multicenter study in Shanghai to evaluate the clinical outcomes and risk factors of EIM with a long-term follow-up from June 1986 to December 2018. The major findings of this study can be summarized as follows: (1) The overall incidence rate of EIM of UC in Shanghai was 11.4\%. (2) Patients with EIM had worse clinical outcomes, which mainly presented as lower remission rates, increased incidence of serious complications, and more likely to develop CRC. (3) A disease duration $>5$ years, an age at diagnosis $>40$ years, refractory clinical symptoms, and moderate or severe anemia contributed to the development of EIM.

The epidemiology of EIM varies widely in different reports, with overall prevalence rates ranging from 6\% to 40\% (Bernstein et al. 2001; Lakatos et al. 2003; Vavricka et al. 2011; Zippi et al. 2014). Maddalena Zippi (Zippi et al. 2014) reported the highest prevalence of $40.6 \%$ in Italy and Charles N. Bernstein (Bernstein et al. 2001) reported the lowest prevalence of $6.2 \%$ in Canada. Moreover, Switzerland, Hungary, and Portugal had prevalence rates of $38.1 \%, 21.3 \%$, and 25.8\% (Lakatos et al. 2003; Vavricka et al. 2011; Veloso et al. 1996), respectively. The over incidence of EIM in Shanghai is only slightly higher than the rate reported in the Canadian retrospective study. These variations may be attributed to specific characteristics of the study populations, differences in the study design and inclusion criteria, accuracy of diagnosis, and previous medical treatment. 
222 In the case of EIM, our results are similar to previous studies (Isene et al. 2015) in that arthritis 223 and oral aphthous ulcers. Interestingly, a previous study in Wuhan, Central China reported a 5.7\% 224 prevalence of EIM in UC (Jiang et al. 2006), which indicated that different regions of the same 225 country may also have different incidence rates of EIM. Taken together, the overall extent of EIM

226

227

228

229

230

231

232

233

234

235

236

238

239

240

241

242

243

244

of UC in China is lower than that in western countries. This may be associated with the different ethnic and environmental factors and it may be beneficial in future studies to determine the genetic and environmental factors involved in the etiology and pathogenesis of EIM.

Despite the lower incidence of EIM in China compared to western countries, EIM can increase the risk of poor clinical outcomes, such as lower remission rates, an increase in serious complications, and even malignant transformation. Therefore, it is imperative for clinicians to uncover the risk factors for the development of EIM earlier and conduct appropriate disease surveillance in clinical practice.

A certain proportion of UC patients presented with serious gastrointestinal symptoms such as diarrhea, stools with blood and mucus, and fecal incontinence. After regular treatment, including the most commonly used 5-ASA (Schroeder et al. 1987), corticosteroid, and immunomodulators, most patients experienced remission, although some patients were still associated with active disease. Although the successful application of anti-TNF- $\alpha$ treatment was a major breakthrough in the treatment of IBD, approximately one-third of patients do not respond to anti-TNF- $\alpha$ treatment, and many others eventually lose responsiveness or become intolerant to these agents (Melmed \& Targan 2010). Noteworthily, previous researches reported that EIM was a flare (Das 1999; Su et al. 2002). As for the characteristic breakdown of intestinal homeostasis in UC, this is considered to arise from a complex interaction of immunological and environmental factors in a genetically predisposed individual. Therefore, we speculated that heterogeneity between individuals led to 
245 different presentations of clinical symptoms, which in turn, could aggravate disease activity 246 towards the development of EIM.

247 The prevalence and severity of anemia are related to the activity of the bowel disorder and a recent 248 meta-analysis reported that anemia occurred in $21 \%$ of UC patients in Europe (Vegh et al. 2016).

249 In this study, a total of 48 patients (17.7\%) experienced moderate anemia; this was slightly lower 250 than the previously reported value of $21 \%$. One reason for this could be because we defined 251 "anemia" as "moderate or severe anemia" with a $\mathrm{Hb}$ level $<90 \mathrm{~g} / \mathrm{L}$. As a retrospective study with 2521158 Crohn's disease (CD) and $1108 \mathrm{UC}$ patients reported, $19.6 \% \mathrm{CD}$ and $21.6 \%$ UC patients were 253 diagnosed with iron deficiency anemia (IDA). In the CD group, the Crohn's disease activity index 254 (CDAI) was considerably higher in patients suffering from IDA, while UC patients with IDA 255 showed a significantly higher rate of erythema nodosum, which is kind of disease activity-related 256 EIM (Madanchi et al. 2018). The results of the current study indicate that anemic patients have a 257 higher disease severity. Furthermore, a previous study demonstrated that anemia in IBD patients 258 was significantly associated with increased ESR, CRP, and CDAI, and that the disease activity scores showed an inverse correlation with the Hb level (Bergamaschi et al. 2010). Taken together, UC patients with moderate anemia usually present with higher disease activity which in turn, aggravates the anemia; it is possible that this interaction is responsible for the development of 262 EIM. There are several limitations to our study. First, it is possible that selection bias was present since all included patients in the present study were diagnosed and managed by colorectal surgeons and gastroenterology clinicians in two different departments. Second, since this is a retrospective study, a loss of follow-up is inevitable. Third, the sample size is relative small, so the conclusion we draw is not accurate enough. A larger sample of multiple-center studies should be imperatively 
268 performed in the near future. On the other hand, there are certain strengths to our study. This study

269 is a retrospective study in Shanghai to evaluate incidence rate of EIM in UC patients. We

270 confirmed the negative effect of EIM on the clinical outcomes. It is more important that we first

271 identified the risk factors associated with EIM in UC, which helps early intervention to prevent

272 the occurrence of EIM.

273

274 Conclusion

275 The present study showed that the incidence rate of EIM of UC in Shanghai was $11.4 \%$.

276 Furthermore, we also discovered the result that EIM was associated with clinical outcomes in UC

277 patients. In addition, a disease duration $>5$ years, an age at diagnosis $>40$ years and refractory

278 clinical symptoms were also found to be contributing factors for the development of EIM. While

279 moderate or severe anemia was associated with EIM. Therefore, appropriate and effective

280 treatment to control active UC and treatment of anemia to improve general condition are

281 imperative in order to prevent the development of EIM and further improve the long-term

282 outcomes of UC patients. 


\section{Reference}

Bergamaschi G, Di Sabatino A, Albertini R, Ardizzone S, Biancheri P, Bonetti E, Cassinotti A, Cazzola P, Markopoulos K, Massari A, Rosti V, Porro GB, and Corazza GR. 2010. Prevalence and pathogenesis of anemia in inflammatory bowel disease. Influence of antitumor necrosis factor-alpha treatment. Haematologica 95:199-205. 10.3324/haematol.2009.009985

Bernstein CN, Blanchard JF, Rawsthorne P, and Yu N. 2001. The prevalence of extraintestinal diseases in inflammatory bowel disease: a population-based study. Am J Gastroenterol 96:1116-1122. 10.1111/j.1572-0241.2001.03756.x

Colia R, Corrado A, and Cantatore FP. 2016. Rheumatologic and extraintestinal manifestations of inflammatory bowel diseases. Ann Med 48:577-585. 10.1080/07853890.2016.1195011

Das KM. 1999. Relationship of extraintestinal involvements in inflammatory bowel disease: new insights into autoimmune pathogenesis. Dig Dis Sci 44:1-13.

Fiocchi C. 1998. Inflammatory bowel disease: etiology and pathogenesis. Gastroenterology $115: 182-205$.

Isene R, Bernklev T, Hoie O, Munkholm P, Tsianos E, Stockbrugger R, Odes S, Palm O, Smastuen M, Moum B, and Group E-IS. 2015. Extraintestinal manifestations in Crohn's disease and ulcerative colitis: results from a prospective, population-based European inception cohort. Scand J Gastroenterol 50:300-305. 10.3109/00365521.2014.991752

Jiang L, Xia B, Li J, Ye M, Yan W, Deng C, Ding Y, Luo H, Hou W, Zhao Q, Liu N, Ren H, Hou X, and Xu H. 2006. Retrospective survey of 452 patients with inflammatory bowel disease in Wuhan city, central China. Inflamm Bowel Dis 12:212-217. 10.1097/01.MIB.0000201098.26450.ae

Lakatos L, Pandur T, David G, Balogh Z, Kuronya P, Tollas A, and Lakatos PL. 2003. Association 
of extraintestinal manifestations of inflammatory bowel disease in a province of western Hungary with disease phenotype: results of a 25-year follow-up study. World $J$ Gastroenterol 9:2300-2307.

Lennard-Jones JE. 1989. Classification of inflammatory bowel disease. Scand J Gastroenterol Suppl 170:2-6; discussion 16-19.

Madanchi M, Fagagnini S, Fournier N, Biedermann L, Zeitz J, Battegay E, Zimmerli L, Vavricka SR, Rogler G, Scharl M, and Swiss IBDCSG. 2018. The Relevance of Vitamin and Iron Deficiency in Patients with Inflammatory Bowel Diseases in Patients of the Swiss IBD Cohort. Inflamm Bowel Dis 24:1768-1779. 10.1093/ibd/izy054

Melmed G, and Targan S. 2010. Future biologic targets for IBD: potentials and pitfalls. Nat Rev Gastroenterol Hepatol 7:110-117.

Podolsky DK. 2002. Inflammatory bowel disease. $N$ Engl $J$ Med 347:417-429. 10.1056/NEJMra020831

Rothfuss KS, Stange EF, and Herrlinger KR. 2006. Extraintestinal manifestations and complications in inflammatory bowel diseases. World J Gastroenterol 12:4819-4831.

SC N. 2017. Understanding and Preventing the Global Increase of Inflammatory Bowel Disease. Gastroenterology 152:313-321.e312.

Schroeder K, Tremaine W, and Ilstrup D. 1987. Coated oral 5-aminosalicylic acid therapy for mildly to moderately active ulcerative colitis. A randomized study. $N$ Engl $J$ Med 317:1625-1629.

Silverberg MS, Satsangi J, Ahmad T, Arnott ID, Bernstein CN, Brant SR, Caprilli R, Colombel JF, Gasche C, Geboes K, Jewell DP, Karban A, Loftus EV, Jr., Pena AS, Riddell RH, Sachar DB, Schreiber S, Steinhart AH, Targan SR, Vermeire S, and Warren BF. 2005. 
331

332

333

334

335

336

338

339

340

341

342

343

344

345

346

347

348

349

350

351

352 353

Toward an integrated clinical, molecular and serological classification of inflammatory bowel disease: report of a Working Party of the 2005 Montreal World Congress of Gastroenterology. Can J Gastroenterol 19 Suppl A:5a-36a.

Su CG, Judge TA, and Lichtenstein GR. 2002. Extraintestinal manifestations of inflammatory bowel disease. Gastroenterol Clin North Am 31:307-327.

Trikudanathan G, Venkatesh PG, and Navaneethan U. 2012. Diagnosis and therapeutic management of extra-intestinal manifestations of inflammatory bowel disease. Drugs 72:2333-2349. 10.2165/11638120-000000000-00000

Vavricka SR, Brun L, Ballabeni P, Pittet V, Prinz Vavricka BM, Zeitz J, Rogler G, and Schoepfer AM. 2011. Frequency and risk factors for extraintestinal manifestations in the Swiss inflammatory bowel disease cohort. Am $J$ Gastroenterol 106:110-119. 10.1038/ajg.2010.343

Vegh Z, Kurti Z, Gonczi L, Golovics PA, Lovasz BD, Szita I, Balogh M, Pandur T, Vavricka SR, Rogler G, Lakatos L, and Lakatos PL. 2016. Association of extraintestinal manifestations and anaemia with disease outcomes in patients with inflammatory bowel disease. Scand $J$ Gastroenterol 51:848-854. 10.3109/00365521.2016.1140807

Veloso FT, Carvalho J, and Magro F. 1996. Immune-related systemic manifestations of inflammatory bowel disease. A prospective study of 792 patients. J Clin Gastroenterol 23:29-34.

Zippi M, Corrado C, Pica R, Avallone EV, Cassieri C, De Nitto D, Paoluzi P, and Vernia P. 2014. Extraintestinal manifestations in a large series of Italian inflammatory bowel disease patients. World J Gastroenterol 20:17463-17467. 10.3748/wjg.v20.146.17463

Peer] reviewing PDF | (2019:01:34655:3:0:NEW 15 May 2019) 
Figure 1

A schematic flow diagram of the present study.

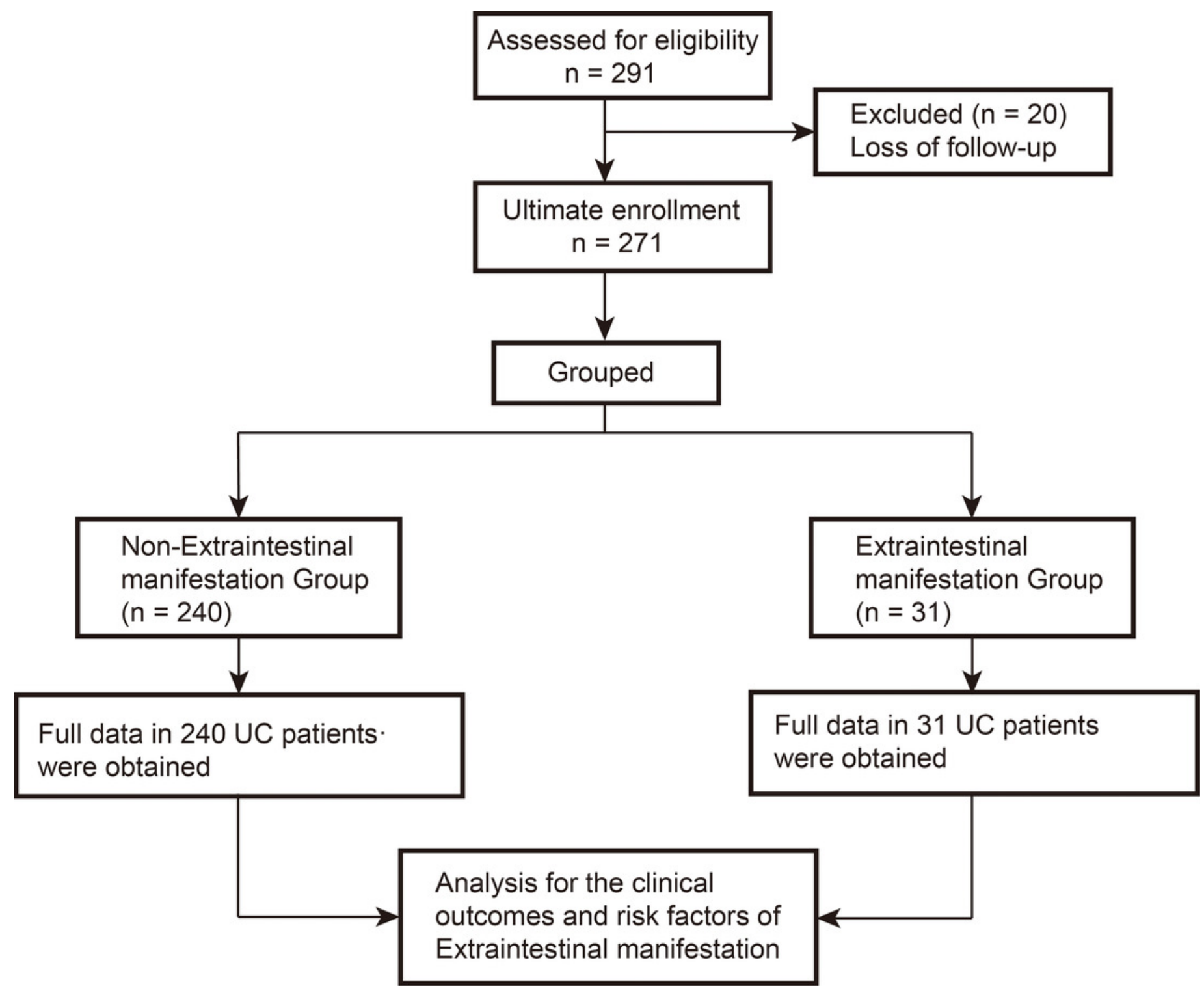




\section{Table $\mathbf{1}$ (on next page)}

Table 1. Patient characteristics at the most recent follow-up visit 
Table 1. Patient characteristics at the most recent follow-up visit

\begin{tabular}{|c|c|c|c|}
\hline Variables & Non-EIM group $(\mathrm{n}=240)$ & EIM group $(n=31)$ & All cases $(n=271)$ \\
\hline Sex (male / female) & $118 / 122$ & $14 / 17$ & $132 / 139$ \\
\hline Age at diagnosis [yr, median (IQR)] & $41.0(28.0-53.0)$ & $47.0(40.0-55.0)$ & $42.0(29.0-53.3)$ \\
\hline Disease duration [yr, median (IQR)] & $6.0(4.0-10.0)$ & $14.0(6.0-17.0)$ & $7.0(4.0-10.0)$ \\
\hline Follow-up time [yr, median (IQR)] & $13.0(9.0-17.0)$ & $9.0(6.0-13.0)$ & $13.0(9.0-17.0)$ \\
\hline \multicolumn{4}{|l|}{ Relapse, n (\%) } \\
\hline First occurrence & $57(23.8)$ & $6(19.4)$ & $63(23.2)$ \\
\hline First recurrence & $47(19.6)$ & $2(6.5)$ & $49(18.1)$ \\
\hline Multiple recurrence & $136(56.7)$ & $23(74.2)$ & $159(58.7)$ \\
\hline Stool with mucous, $\mathrm{n}(\%)$ & $112(46.7)$ & $21(67.7)$ & $133(49.1)$ \\
\hline Bloody stool, n (\%) & $207(86.3)$ & $25(80.6)$ & $232(85.6)$ \\
\hline Abdominal bloating and distension, $\mathrm{n}(\%)$ & $48(20.0)$ & $9(29.0)$ & $57(21.0)$ \\
\hline Diarrhea & $159(66.3)$ & $28(90.3)$ & $187(69.0)$ \\
\hline \multicolumn{4}{|l|}{ Stool frequency, n (\%) } \\
\hline$<4$ times & $204(85.0)$ & $18(58.1)$ & $222(81.9)$ \\
\hline$\geqq 4$ time & $36(15.0)$ & $13(41.9)$ & $49(18.1)$ \\
\hline Weight loss, n (\%) & $86(35.8)$ & $16(51.6)$ & $102(37.6)$ \\
\hline Extraintestinal manifestations (EIMs), $\mathrm{n}(\%)$ & & $31(11.4)$ & $31(11.4)$ \\
\hline Single EIMs & & $23(8.4)$ & $23(8.4)$ \\
\hline Multiple EIMs & & $8(3.0)$ & $8(3.0)$ \\
\hline History of surgery, n (\%) & $30(12.5)$ & $7(22.6)$ & $37(13.7)$ \\
\hline Family history, n (\%) & $15(6.3)$ & $3(9.7)$ & $18(6.6)$ \\
\hline Family history of CRC & $3(1.3)$ & $1(3.2)$ & $4(1.5)$ \\
\hline Family history of IBD & $2(0.8)$ & $1(3.2)$ & $3(1.1)$ \\
\hline Family history of other caner & $12(5.0)$ & $1(3.2)$ & $13(4.8)$ \\
\hline \multicolumn{4}{|l|}{ Extent of UC, n (\%) } \\
\hline E1 & $6(2.5)$ & $0(0.0)$ & $6(2.2)$ \\
\hline E2 & $125(52.1)$ & $16(51.6)$ & $141(52.0)$ \\
\hline E3 & $109(45.4)$ & $15(46.9)$ & $124(45.8)$ \\
\hline Probiotics, n (\%) & $84(35.0)$ & $11(35.5)$ & $95(35.1)$ \\
\hline Steroids, n (\%) & $148(61.7)$ & $21(67.7)$ & $169(62.4)$ \\
\hline Immunomodulators, $\mathrm{n}(\%)$ & $32(13.3)$ & $2(6.5)$ & $34(12.5)$ \\
\hline $\mathrm{Hb}(\mathrm{g} / \mathrm{L}$, mean $\pm \mathrm{SD})$ & $114.5 \pm 25.1$ & $109.7 \pm 27.1$ & $113.9 \pm 25.3$ \\
\hline $\operatorname{ALT}(\mathrm{g} / \mathrm{L}$, mean $\pm \mathrm{SD})$ & $28.6 \pm 23.1$ & $24.7 \pm 11.6$ & $28.2 \pm 22.1$ \\
\hline
\end{tabular}

1 CRC: colorectal cancer, UC: ulcerative colitis, IQR: interquartile range, Hb: hemoglobin, IBD: inflammatory 2 bowel disease, Alb: Albumin 
Table 2 (on next page)

Analysis of extraintestinal manifestations (EIM) 
Table 2. Analysis of extraintestinal manifestations (EIM)

\begin{tabular}{llll}
\hline EIM & Non-EIM group (\%) & EIM group (\%) & N (\%) \\
\hline Rash & $0(0.0)$ & $7(22.6)$ & $7(2.6)$ \\
Oral aphthous ulcers & $0(0.0)$ & $10(32.3)$ & $10(3.7)$ \\
Arthritis & $0(0.0)$ & $14(45.2)$ & $14(5.2)$ \\
Primary sclerosing cholangitis (PSC) & $0(0.0)$ & $2(6.5)$ & $2(0.7 \%)$ \\
Iritis & $0(0.0)$ & $1(3.2)$ & $1(0.4)$ \\
Vasculitis & $0(0.0)$ & $1(3.2)$ & $1(0.4)$ \\
Ankylosing spondylitis & $0(0.0)$ & $1(3.2)$ & $1(0.4)$ \\
Autoimmune hepatitis & $0(0.0)$ & $1(3.2)$ & $1(0.4)$ \\
Glomerulonephritis & $0(0.0)$ & $2(6.5)$ & $2(0.7)$ \\
\hline
\end{tabular}

1 
Table 3 (on next page)

Analysis of complications in ulcerative colitis (UC) patients. 
Table 3. Analysis of complications in ulcerative colitis (UC) patients.

\begin{tabular}{llll}
\hline Complications & Non-EIM group (\%) & EIM group (\%) & N (\%) \\
\hline Colorectal stricture & $28(11.7)$ & $8(25.8)$ & $36(13.3)$ \\
Bleeding & $24(10.0)$ & $4(12.9)$ & $28(10.3)$ \\
Inflammatory polyps & $57(23.8)$ & $6(19.4)$ & $63(23.2)$ \\
Intestinal obstruction & $16(6.7)$ & $5(16.1)$ & $21(7.7)$ \\
Colon perforation & $5(2.1)$ & $1(3.2)$ & $6(2.2)$ \\
Toxic megacolon & $2(0.8)$ & $0(0.0)$ & $2(0.7)$ \\
Abscess formation & $3(1.3)$ & $0(0.0)$ & $3(1.1)$ \\
CRC & $7(2.9)$ & $4(12.9)$ & $11(4.1)$ \\
\hline
\end{tabular}

1 CRC: colorectal cancer; EIM: extraintestinal manifestation 


\section{Table 4 (on next page)}

Comparison of clinical outcomes between ulcerative colitis patients with and without extraintestinal manifestations (EIMs). 
Table 4. Comparison of clinical outcomes between ulcerative colitis patients with and without extraintestinal manifestations (EIMs)

\begin{tabular}{llll}
\hline Variables & Non-EIM group & EIM group & p value \\
\cline { 2 - 3 } Remission, n (\%) & $113(47.1)$ & $23(74.2)$ & $0.04^{\mathrm{a}}$ \\
No & $127(52.9)$ & $8(25.8)$ & \\
Yes & & & $0.035^{\mathrm{a}}$ \\
Serious complications, n (\%) & $200(83.3)$ & $21(67.7)$ & \\
No & $40(16.7)$ & $10(32.3)$ & \\
Yes & & & $0.160^{\mathrm{a}}$ \\
Surgery, n (\%) & $150(62.5)$ & $19(61.3)$ & \\
No & $90(37.5)$ & $12(38.7)$ & \\
Yes & & & $0.030^{\mathrm{b}}$ \\
CRC, n (\%) & $233(97.1)$ & $27(87.1)$ & \\
No & $7(2.9)$ & $4(12.9)$ & \\
Yes & & & $0.718^{\mathrm{b}}$ \\
Death, n (\%) & $232(96.7)$ & $29(93.5)$ & \\
No & $8(3.3)$ & $2(6.5)$ & \\
Yes & & \\
\hline
\end{tabular}

1 CRC: colorectal cancer, a: Chi-squared; b: Fisher's exact test. Serious complications: Intestinal obstruction, 2 colon perforation and gastrointestinal bleeding 


\section{Table 5 (on next page)}

Risk factors for extraintestinal manifestations (EIMs) in ulcerative colitis patients. 
Table 5. Risk factors for extraintestinal manifestations (EIMs) in ulcerative colitis patients

\begin{tabular}{|c|c|c|c|}
\hline Variables & Non-EIM group & EIM group & $\mathrm{p}$ value \\
\hline Sex, n $(\%)$ & & $\square$ & $0.675^{\mathrm{a}}$ \\
\hline Male & $118(49.2)$ & $14(45.2)$ & \\
\hline Female & $122(50.8)$ & $17(54.8)$ & \\
\hline Age at diagnosis, $\mathrm{n}(\%)$ & & & $0.011^{\mathrm{a}}$ \\
\hline$<40 \mathrm{y}$ & $112(46.7)$ & $7(22.6)$ & \\
\hline$\geqq 40 \mathrm{y}$ & $128(53.3)$ & $24(77.4)$ & \\
\hline Disease duration, $\mathrm{n}(\%)$ & & & $0.013^{\mathrm{a}}$ \\
\hline$<5 y$ & $84(35)$ & $4(12.9)$ & \\
\hline$\geqq 5 \mathrm{y}$ & $156(65)$ & $27(87.1)$ & \\
\hline Relapse, n (\%) & & & $0.117^{\mathrm{b}}$ \\
\hline First occurrence & $57(23.8)$ & $6(19.4)$ & \\
\hline First recurrence & $47(19.6)$ & $2(6.5)$ & \\
\hline Multiple recurrence & $136(56.7)$ & $23(74.2)$ & \\
\hline Refractory clinical symptoms, $\mathrm{n}(\%)$ & & & $<0.001^{\mathrm{a}}$ \\
\hline No & $204(85.0)$ & $18(58.1)$ & \\
\hline Yes & $36(15.0)$ & $13(41.9)$ & \\
\hline Weight loss, $\mathrm{n}(\%)$ & & & $0.088^{\mathrm{a}}$ \\
\hline No & $154(64.2)$ & $15(48.4)$ & \\
\hline Yes & $86(35.8)$ & $16(51.6)$ & \\
\hline History of surgery, n (\%) & & & $0.208^{a}$ \\
\hline No & $210(87.5)$ & $24(77.4)$ & \\
\hline Yes & $30(12.5)$ & $7(22.6)$ & \\
\hline Family history, $\mathrm{n}(\%)$ & & & $0.346^{\mathrm{a}}$ \\
\hline No & $225(93.8)$ & $28(90.3)$ & \\
\hline Family history of CRC or IBD & $5(2.1)$ & $2(6.5)$ & \\
\hline Family history of other cancers & $10(4.2)$ & $1(3.2)$ & \\
\hline Extent of UC, n (\%) & & & $0.690^{\mathrm{b}}$ \\
\hline E1 & $6(2.5)$ & $0(0.0)$ & \\
\hline E2 & $124(51.7)$ & $16(51.6)$ & \\
\hline E3 & $110(45.8)$ & $15(48.4)$ & \\
\hline Steroids, n (\%) & & & $0.511^{\mathrm{a}}$ \\
\hline No & $92(38.3)$ & $10(32.3)$ & \\
\hline Yes & $148(61.7)$ & $21(67.7)$ & \\
\hline Immunomodulators, $\mathrm{n}(\%)$ & & & $0.423^{\mathrm{a}}$ \\
\hline No & $208(86.7)$ & $29(93.5)$ & \\
\hline Yes & $32(13.3)$ & $2(6.5)$ & \\
\hline $\mathrm{Hb}, \mathrm{n}(\%)$ & & & $0.024^{\mathrm{a}}$ \\
\hline$\geqq 90 \mathrm{~g} / \mathrm{L}$ & $202(84.2)$ & $21(67.7)$ & \\
\hline
\end{tabular}




\begin{tabular}{clll}
$<90 \mathrm{~g} / \mathrm{L}$ & $38(15.8)$ & $10(32.3)$ & \\
$\begin{array}{c}\text { Alb }, \mathrm{n}(\%) \\
\geqq 35 \mathrm{~g} / \mathrm{L}\end{array}$ & $90(37.5)$ & $8(25.8)$ & $0.202^{\mathrm{a}}$ \\
$<35 \mathrm{~g} / \mathrm{L}$ & $150(62.5)$ & $23(74.2)$ & \\
\hline
\end{tabular}

1 CRC: colorectal cancer, UC: ulcerative colitis, $\mathrm{Hb}$ : hemoglobin, IBD: inflammatory bowel disease, Alb: 2 Albumin, a: Chi-squared or Fisher's exact test. b: Wilcoxon's rank-sum test.

3 


\section{Table 6(on next page)}

Multivariate logistic regression analysis of risk factors for extraintestinal manifestations (EIMs) in ulcerative colitis patients. 
Table 6. Multivariate logistic regression analysis of risk factors for extraintestinal manifestations (EIMs) in ulcerative colitis patients

\begin{tabular}{lllllll}
\hline \multirow{2}{*}{ Variable } & \multicolumn{3}{c}{ Univariate $^{\mathrm{a}}$} & \multicolumn{3}{c}{ Multivariate $^{\mathrm{b}}$} \\
\cline { 2 - 7 } & Odds Ratio & $95 \% \mathrm{CI}$ & $\mathrm{p}$ value & Odds Ratio & $95 \%$ CI & $\mathrm{p}$ value \\
\hline Disease duration $(\geqq 5 \mathrm{y})$ & 3.635 & $1.231-10.735$ & 0.020 & 3.721 & $1.209-11.456$ & 0.022 \\
Age at diagnosis $(\geqq 40 \mathrm{y})$ & 3.000 & $1.245-7.228$ & 0.014 & 2.924 & $1.165-7.340$ & 0.022 \\
Refractory clinical symptoms & 4.093 & $1.875-9.077$ & 0.001 & 4.119 & $1.758-9.650$ & 0.001 \\
Moderate anemia & 2.531 & $1.105-5.799$ & 0.028 & 2.592 & $1.047-6.413$ & 0.039 \\
Relapse & 1.418 & $0.858-2.342$ & 0.173 & $\square$ & $\square$ & $\square$ \\
\hline
\end{tabular}

CI: confidence interval, a: Univariate logistic regression analysis, b: Multivariate logistic regression analysis. 